\title{
COMPLETE RESIDUE SYSTEMS IN THE RING OF MATRICES OF RATIONAL INTEGERS
}

\author{
JAU-SHYONG SHIUE \\ Department of Mathematical Sciences \\ National Chengchi University \\ Taipei, Taiwan \\ Republic of China \\ and
}

\section{CHIE-PING HWANG}

Department of Mathematics

National Central University

Chung1i, Taiwan

Republic of China

(Received April 1, 1977 and in revised form August 29, 1977)

ABSTRACT. This paper deals with the characterizations of the complete residue system mod. $G$, where $G$ is any $n \times n$ matrix, in the ring of $n \times n$ matrices.

KEY WORDS AND PHRASES. Complete residue system, ring of Gaussian integers, representations for the complete residue system.

AMS(MOS) SUBJECT CLASSIFICATION (1970) CODES. 12F05, $12 B 35$.

1. INTRODUCTION.

Let $Z$ denote the ring of rational integers and $Z(i)$ be the ring of 
Gaussian integers. Jordan and Potratz [1] have exhibited several representations for the complete residue system (in short, C.R.S.) mod.r. in the ring of Gaussian integers. Also it is well known that the ring of Gaussian integers is isomorphic to the ring of $2 \times 2$ matrices of the form $\left(\begin{array}{rr}a & b \\ -b & a\end{array}\right)$, $a, b$ in $z$. This raises the question of characterizing the C.R.S. mod. G, where $G$ is any $n \times n$ matrix, in the ring of $n \times n$ matrices of which we denote by $\operatorname{Mat}_{n}(Z)$.

2. THE COMPLETE RESIDUE SYSTEM IN Mat ${ }_{n}(Z)$.

First of all, we define $A \mid B$ mean there is a matrix $C$ such that $B=C A$, and $A \equiv B$ mod. $U$ means that $U \mid A-B$. Now we can give a definition of the C.R.S. mod. $U$ in the ring of $\operatorname{Mat}_{n}(Z)$.

DEFINITION. Let $U$ be in $\operatorname{Mat}_{n}(Z)$ with $\operatorname{det} U \neq 0$. Then a subset $J$ of Mat ${ }_{n}(Z)$ is called a C.R.S. mod. $U$ if and only if for any $A$ in Mat ${ }_{n}(Z)$ there exists uniquely a matrix $B$ in $\mathrm{J}$ such that $\mathrm{A} \equiv \mathrm{B} \bmod$. $\mathrm{U}$.

LEMMA 1. Let $G=\operatorname{diag}\left(g_{1}, g_{2}, \ldots, g_{n}\right)$ with $g_{i} \neq 0, i=1,2, \ldots, n$. Let $E_{i j}$ be the matrix units, then

$I_{i k}=\left\{a \varepsilon Z: G \mid \sum_{m=i}^{n} \sum_{j=1}^{n} a_{m j} E_{m j}\right.$ where $a_{m j}$ in $\left.z, a_{i 1}=a_{i 2}=\ldots=a_{i k-1}=0, a_{i k}=a\right\}$

are the principal ideals generated by a positive integer $g_{k}$, where $1, k=1,2, \ldots, n$.

PROOF. It is clear the $I_{i k}$ are ideals in $Z$. But $Z$ is a P.I.D., therefore $I_{i k}$ are principal ideals generated by a positive integer $d_{i k}$. Since $g_{k} E_{i k}=E_{i k} G$, then $g_{k}$ is in $I_{i k}$, i.e., $d_{i k} \mid g_{k}$. On the other hand, for $d_{i k}$ in $I_{i k}$ we have $\sum_{m=1}^{n} \sum_{j=1}^{n} a_{m j} E_{m j}=\left(t_{1 k}\right) G$ for some $\left(t_{i k}\right)$, where $a_{m j}$ is in $z$, $a_{i 1}=a_{12}=\ldots=a_{1 k-1}=0, a_{i k}=d_{1 k}$. It follows that $d_{i k}=t_{1 k} g_{k}$, 1.e., $d_{1 k}=\left|g_{k}\right|$. This completes the proof. 
LEMMA 2. Let $G=\operatorname{diag}\left(g_{1}, g_{2}, \ldots, g_{n}\right)$ with $g_{k} \neq 0, k=1,2, \ldots, n$. Then $J=\left\{\left(r_{i k}\right): 0 \leq r_{i k}<\left|g_{k}\right|, i, k=1,2, \ldots, n\right\}$ forms a complete residue system mod. G.

PROOF. (1) For any $A=\left(a_{i k}\right)$ in Mat ${ }_{n}(z)$, there exist $p_{i k}, r_{i k}$ in $Z$ such that $a_{i k}=p_{i k}\left|g_{k}\right|+r_{i k}$, where $0 \leq r_{i k}<\left|g_{k}\right|$. Therefore $A-\left(p_{i k} \cdot\left|g_{k}\right|\right)=\left(r_{i k}\right)$. But $\left|g_{k}\right| \cdot E_{i k}=\left|g_{k}\right| \cdot g_{k}^{-1} E_{i k} G$, and therefore G $\mid A-\left(r_{i k}\right)$. This shows that $A \equiv\left(r_{i k}\right) \bmod . G$.

(2) If $\left(r_{i k}\right) \equiv\left(s_{i k}\right) \bmod . G$, where $0 \leq r_{i k}, s_{i k}<\left|g_{k}\right|$, then G $\mid\left(r_{i k}-s_{i k}\right)$, i.e., $r_{i 1}-s_{11}$ is in $I_{11}$ (by Lemma 1 ). This implies that $g_{1} \mid\left(r_{11}-s_{11}\right)$, and so $r_{11}=s_{11}$, for $0 \leq\left|r_{11}-s_{11}\right|<\left|g_{1}\right|$. It follows that $r_{12}-s_{12}$ is in $I_{12}$. Therefore $g_{2} \mid\left(r_{12}-s_{12}\right)$ and $r_{12}=s_{12}$, for $0 \leq\left|r_{12}-s_{12}\right|<\left|g_{2}\right|$. Continuing in this way, we must have $r_{i k}=s_{1 k}$, for all $1, k=1,2, \ldots, n$.

THEOREM 1. If $G$ is a $n \times n$ matrix with $\operatorname{det} G \neq 0$, and if $U$ and $V$ are unimodular $n \times n$ matrices such that $U G V=\operatorname{diag}\left(g_{1}, g_{2}, \ldots, g_{n}\right)$, then $J=\left\{\left(r_{i k}\right) v^{-1}: 0 \leq r_{i k}<\left|g_{k}\right|, 1, k=1,2, \ldots, n\right\}$ forms a complete residue system mod. G.

PROOF. (1) By Lemma 2, for any $n \times n$ matrix $A$, there exists a matrix $\left(r_{1 k}\right)$ with $0 \leq r_{1 k}<\left|g_{k}\right|$ such that $A V \equiv\left(r_{1 k}\right)$ mod. UGV., i.e., $A \equiv\left(r_{1 k}\right) V^{-1} \bmod . G$.

(2) Let $\left(r_{i k}\right) v^{-1} \equiv\left(s_{i k}\right) v^{-1} \bmod$. $G$, where $0 \leq r_{i k}, s_{i k}<\left|g_{k}\right|$. It follows that $\left(r_{i k}\right) \equiv\left(s_{i k}\right)$ mod. UGV. Therefore $\left(r_{i k}\right)=\left(s_{i k}\right)$.

COROLLARY 1. If $J$ forms a C.R.S. mod. $G$, and $U$ and $V$ are unimodular $n \times n$ matrices, then \{URV : $R$ in J\} forms a C.R.S. mod. GV.

COROLLARY 2. If $G$ is a $n \times n$ matrix with $\operatorname{det} G \neq 0$, then the cardinality of the C.R.S. $\bmod . \mathrm{G}$ is $|\operatorname{det} G|^{\mathrm{n}}$. 
3. THE COMPLETE RESIDUE SYSTEM IN $\mathrm{Mat}_{2}(\mathrm{Z})$.

By restricting the order of the matrix we may relax the condition on the diagonable matrix.

LEMMA 3. Let $U=\left(\begin{array}{ll}u_{11} & u_{12} \\ u_{21} & u_{22}\end{array}\right) \varepsilon \operatorname{Mat}_{2}(z)$ with $\operatorname{det} U \neq 0$, then

(1) $I_{0}=\left\{\begin{array}{lllll}a & \varepsilon & Z & : U & \mid\left(\begin{array}{ll}a & \alpha \\ \beta & r\end{array}\right) \text { for some } \alpha, \beta, r \in Z\end{array}\right\}$ and

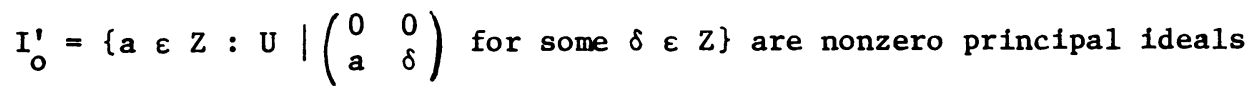

of $\mathrm{Z}$ generated by a positive integer $d=$ g.c.d. $\left(u_{1}, u_{2}\right)$. Moreover $I_{0}=I_{0}^{\prime}$.

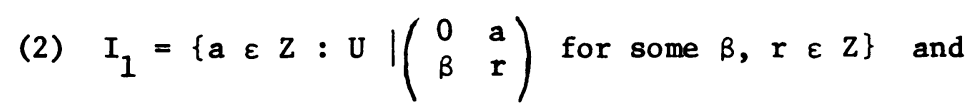

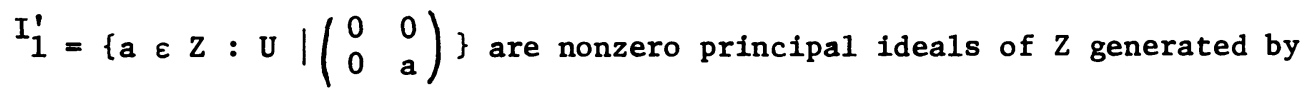

a positive integer $\frac{\mid \text { det } U \mid}{d}$. Moreover, $I_{1}=I_{1}^{\prime}$.

PROOF. (1) a $\varepsilon I_{0}$ implies $U \mid\left(\begin{array}{ll}a & \alpha \\ \beta & r\end{array}\right)$ for some $\alpha, \beta, r \varepsilon z$, and then $\mathrm{U} \mid\left(\begin{array}{ll}0 & 0 \\ 1 & 0\end{array}\right)\left(\begin{array}{ll}a & \alpha \\ \beta & r\end{array}\right)=\left(\begin{array}{ll}0 & 0 \\ a & \alpha\end{array}\right)$, i.e., a $\varepsilon I_{0}^{\prime}$. This shows that $I_{0} \subseteq I_{0}^{\prime}$. On the other hand, $b \in I_{0}^{\prime}$ implies $U \mid\left(\begin{array}{ll}0 & 0 \\ b & \delta\end{array}\right)$ for some $\delta \varepsilon z$ and then $\mathrm{U} \mid\left(\begin{array}{ll}0 & 1 \\ 0 & 0\end{array}\right)\left(\begin{array}{ll}0 & 0 \\ \mathrm{~b} & \delta\end{array}\right)=\left(\begin{array}{ll}\mathrm{b} & \delta \\ 0 & 0\end{array}\right)$, i.e., $\mathrm{b} \varepsilon \mathrm{I}_{\mathrm{o}}$. Therefore $I_{0}=I_{0}^{\prime}$. It is clear that $I_{0}$ is an ideal of $Z$. Now $\operatorname{det} U \varepsilon I_{0}$, for $U \mid\left(\begin{array}{cl}\operatorname{det} U & 0 \\ 0 & \operatorname{det} U\end{array}\right)$.

Thus $I_{0}$ is a nonzero ideal of $Z$. But $Z$ is a P.I.D., therefore $I_{0}$ is an Ideal generated by a positive integer d. Since $U \mid U$ implies $U \mid\left(\begin{array}{ll}0 & 1 \\ 0 & 0\end{array}\right) U=\left(\begin{array}{cc}U_{21} & U_{22} \\ 0 & 0\end{array}\right)$, we have $U_{11}, U_{12} \varepsilon I_{0}$, and then $d\left|U_{11}, d\right| U_{21}$. By $d \varepsilon I_{0}$, we have $U^{-} \mid\left(\begin{array}{ll}0 & 0 \\ d & \delta\end{array}\right)$, i.e., $\left(\begin{array}{ll}0 & 0 \\ d & \delta\end{array}\right)=\left(\begin{array}{ll}t_{11} & t_{12} \\ t_{21} & t_{22}\end{array}\right) U$ for some $\left(\begin{array}{ll}t_{11} & t_{12} \\ t_{21} & t_{22}\end{array}\right) \varepsilon \operatorname{Mat}_{2}(z)$.

Therefore $d=t_{21} U_{11}+t_{22} U_{21}$. If $x \mid U_{11}$ and $x \mid U_{21}$, then $x \mid d$. Thus $d=g \cdot c \cdot d \cdot\left(U_{11}, U_{21}\right)$. 
(2) a $\varepsilon \quad I_{1}$ implies $U \mid\left(\begin{array}{ll}0 & a \\ \beta & r\end{array}\right)$ for some $\beta, r \varepsilon z$ and then $\mathrm{U} \mid\left(\begin{array}{ll}0 & 0 \\ 1 & 0\end{array}\right)\left(\begin{array}{ll}0 & a \\ B & r\end{array}\right)=\left(\begin{array}{ll}0 & 0 \\ 0 & a\end{array}\right)$, i.e., a $\varepsilon I_{1}^{\prime}$. Thus $I_{1} \subseteq I_{1}^{\prime}$. Conversely, if $b \in I_{1}^{\prime}$, then $U \mid\left(\begin{array}{ll}0 & 0 \\ 0 & b\end{array}\right)$ and so $U \mid\left(\begin{array}{ll}0 & 1 \\ 0 & 0\end{array}\right)\left(\begin{array}{ll}0 & 0 \\ 0 & b\end{array}\right)=\left(\begin{array}{ll}0 & b \\ 0 & 0\end{array}\right)$, 1.e., $b \varepsilon I_{1}$. It is also clear that $I_{1}$ is an ideal of $Z$. Now $\frac{\operatorname{det} U}{d} \varepsilon I_{1}$ for all $U$ such that $\left(\begin{array}{cc}0 & 0 \\ 0 & \frac{\operatorname{det} U}{d}\end{array}\right)=\left(\begin{array}{cc}0 & 0 \\ \frac{-u_{21}}{d} & \frac{u_{12}}{d}\end{array}\right) U$, and then $I_{1}$ is a nonzero ideal of Z. But $Z$ is a P.I.D., and then $I_{1}$ is an ideal generated by a positive integer g. Now $\frac{\operatorname{det} U}{d} \varepsilon I_{1}$ implies $\frac{\operatorname{det} U}{d} \varepsilon I_{1}$, i.e., $g \mid \frac{|\operatorname{det} U|}{d}$. By $g \varepsilon I_{1}$, we have $\mathrm{U} \mid\left(\begin{array}{ll}0 & 0 \\ 0 & \mathrm{~g}\end{array}\right)$, i.e., $\operatorname{det} \mathrm{U} \mid\left(\begin{array}{ll}0 & 0 \\ 0 & \mathrm{~g}\end{array}\right)\left(\begin{array}{cc}\mathrm{u}_{22} & -\mathrm{u}_{12} \\ -\mathrm{u}_{21} & \mathrm{u}_{11}\end{array}\right)=\left(\begin{array}{cc}0 & 0 \\ -g \mathrm{u}_{21} & g u_{11}\end{array}\right)$, and then $\operatorname{det} U \mid \mathrm{gU}_{21}, \operatorname{det} \mathrm{|} \mathrm{gU}_{11}$.

By the proof of (1), we have $d=t_{21} U_{11}+t_{22} U_{21}$, and then $g d=t_{21}\left(g U_{11}\right)+t_{22}\left(g u_{21}\right)$ or $\frac{|\operatorname{det} U|}{d} \mid g$. Therefore $g=\frac{|\operatorname{det} U|}{d}$. This completes the proof of (2). THEOREM 2. Let $U=\left(\begin{array}{ll}u_{11} & u_{12} \\ u_{21} & u_{22}\end{array}\right) \varepsilon \operatorname{Mat}_{2}(z)$ with $\operatorname{det} U \neq 0$, let $d=g \cdot c \cdot d \cdot\left(u_{11}, u_{21}\right)$. Then $J=\left\{R=\left(\begin{array}{ll}r_{11} & r_{12} \\ r_{21} & r_{22}\end{array}\right) \varepsilon \operatorname{Mat}_{2}(z): 0 \leq r_{11}\right.$, $\left.r_{21}<d, 0 \leq r_{12}, r_{22}<\frac{|\operatorname{det} U|}{d}\right\}$ is a complete residue system (mod. U) in $\operatorname{Mat}_{2}(\mathrm{Z})$.

PROOF. (1) From $d \varepsilon I_{0}, \frac{|\operatorname{det} U|}{d} \varepsilon I_{1}$, we have $\mathrm{U}\left|\left(\begin{array}{ll}\mathrm{d} & \alpha \\ \beta & \mathrm{r}\end{array}\right), \mathrm{U}\right|\left(\begin{array}{ll}0 & 0 \\ \mathrm{~d} & \eta\end{array}\right), \mathrm{U}\left|\left(\begin{array}{cc}0 & \frac{\mid \operatorname{det} \mathrm{d}}{\mathrm{d}} \\ \varepsilon & \delta\end{array}\right), \mathrm{U}\right|\left(\begin{array}{cc}0 & 0 \\ 0 & \frac{|\operatorname{det} \mathrm{U}|}{\mathrm{d}}\end{array}\right)$, i.e., 
there exists $T_{i} \in \operatorname{Mat}_{2}(Z), i=1,2,3,4$ such that

$\left(\begin{array}{ll}\mathrm{d} & \alpha \\ \beta & \mathrm{r}\end{array}\right)=\mathrm{T}_{1} \mathrm{U},\left(\begin{array}{cc}0 & \frac{|\operatorname{det} U|}{\mathrm{d}} \\ \varepsilon & \delta\end{array}\right)=\mathrm{T}_{2} \mathrm{U},\left(\begin{array}{cc}0 & 0 \\ \mathrm{~d} & \mathrm{n}\end{array}\right)=\mathrm{T}_{3} \mathrm{U},\left(\begin{array}{cc}0 & 0 \\ 0 & \frac{|\operatorname{det} \mathrm{U}|}{\mathrm{d}}\end{array}\right)=\mathrm{T}_{4} \mathrm{U}$.

For any matrix $A=\left(\begin{array}{cc}a_{11} & a_{12} \\ a_{21} & a_{22}\end{array}\right) \varepsilon \operatorname{Mat}_{2}(z)$, there exists $p_{11}, r_{11} \varepsilon Z$ such that $a_{11}=p_{11} d+r_{11}$ where $0 \leq r_{11}<d$. Thus $A-p_{11} T_{1} U=\left(\begin{array}{ll}r_{11} & b_{12} \\ b_{21} & b_{22}\end{array}\right)$, for some $b_{12}, b_{21}, b_{22} \varepsilon z$. Moreover, $b_{12}=P_{12} \frac{|\operatorname{det} U|}{d}+r_{12}$ for some $\mathrm{p}_{12}, \mathrm{r}_{12} \in \mathrm{z}, 0 \leq \mathrm{r}_{12}<\frac{|\operatorname{det} \mathrm{U}|}{\mathrm{d}}$. Then $\mathrm{A}-\mathrm{p}_{11} \mathrm{~T}_{1} \mathrm{U}-\mathrm{p}_{12} \mathrm{~T}_{2} \mathrm{U}=\left(\begin{array}{ll}\mathrm{r}_{11} & \mathrm{r}_{12} \\ \mathrm{c}_{21} & \mathrm{c}_{22}\end{array}\right)$ for some $c_{21}, c_{22} \varepsilon z$. Again $c_{21}=p_{21}-d+r_{21}$ for some $p_{21}, r_{21} \varepsilon z$, $0 \leq \mathrm{r}_{21}<\mathrm{d}$. Then $\mathrm{A}-\mathrm{p}_{11} \mathrm{~T}_{1} \mathrm{U}-\mathrm{p}_{12} \mathrm{~T}_{2} \mathrm{U}-\mathrm{p}_{21} \mathrm{~T}_{3} \mathrm{U}=\left(\begin{array}{ll}\mathrm{r}_{11} & \mathrm{r}_{12} \\ \mathrm{r}_{21} & \mathrm{r}_{22}\end{array}\right)$ for some $\mathrm{d}_{22} \varepsilon$ z. Finally $\mathrm{d}_{22}=\mathrm{p}_{22} \frac{|\operatorname{det} \mathrm{U}|}{\mathrm{d}}+\mathrm{r}_{22}$ for some $\mathrm{p}_{22}, \mathrm{r}_{22} \varepsilon \mathrm{z}, 0 \leq \mathrm{r}_{22}<\frac{|\operatorname{det} \mathrm{U}|}{\mathrm{d}}$, implies $A-p_{11} T_{1} U-p_{12} T_{2} U-p_{21} T_{3} U-p_{22} T_{4} U=\left(\begin{array}{cc}r_{11} & r_{12} \\ r_{21} & r_{22}\end{array}\right)$ or $\mathrm{U} \mid \mathrm{A}-\left(\begin{array}{ll}\mathrm{r}_{11} & \mathrm{r}_{12} \\ \mathrm{r}_{21} & \mathrm{r}_{22}\end{array}\right)$, where $0 \leq \mathrm{r}_{11}, \mathrm{r}_{21}<\mathrm{d}, 0 \leq \mathrm{r}_{22}, \mathrm{r}_{12}<\frac{|\operatorname{det} \mathrm{U}|}{\mathrm{d}}$. This proves that for any matrix $A \varepsilon \operatorname{Mat}_{2}(Z)$ there exists $R \varepsilon J_{2}$ such that $A \equiv R(\bmod . U)$.

(2) Assume that $\left(\begin{array}{ll}r_{11} & r_{12} \\ r_{21} & r_{22}\end{array}\right) \equiv\left(\begin{array}{ll}s_{11} & s_{12} \\ s_{21} & s_{22}\end{array}\right) \quad$ (mod. U) where $0 \leq r_{11}, r_{21}, s_{11}, s_{21}<d, 0 \leq r_{12}, r_{22}, s_{12}, s_{22}<\frac{|\operatorname{det} U|}{d}$. 
This implies

$U \mid\left(\begin{array}{ll}r_{11}-s_{11} & r_{12^{-s}} 12 \\ r_{21}-s_{21} & r_{22^{-s}}\end{array}\right)$, i.e., $r_{11}-s_{11} \varepsilon I_{0}$, or $d \mid r_{11}-s_{11}$.

Now $0 \leq\left|r_{11}-s_{11}\right|<d, \quad r_{11}=s_{11}$. It follows that $U\left(\begin{array}{ll}0 & r_{12^{-s}} \\ r_{21}-s_{21} & r_{22}-s_{22}\end{array}\right)$, 1.e., $r_{12}-s_{12} \varepsilon I_{1}$, or $\frac{|\operatorname{det} U|}{d} \mid\left(r_{12}-s_{12}\right)$. But $0 \leq\left|r_{12}-s_{12}\right|<\frac{|\operatorname{det} U|}{d}$, so that $r_{12}=s_{12}$.

It follows that

$\mathrm{U}\left\lceil\left(\begin{array}{ll}0 & 0 \\ r_{21}-s_{21} & r_{22}-s_{22}\end{array}\right)\right.$, i.e., $r_{21}-s_{21} \varepsilon I_{0}$ or d $\mid\left(r_{21}-s_{21}\right)$. Also $0 \leq\left|r_{21}-s_{21}\right|<d$, so that $r_{21}=s_{21}$. This implies that $U \mid\left(\begin{array}{ll}0 & 0 \\ 0 & r_{22}-s_{22}\end{array}\right)$, i.e., $r_{22}-s_{22} \varepsilon I_{1}$ or $\frac{|\operatorname{det} U|}{\mathrm{d}} \mid\left(r_{22}-s_{22}\right)$. Finally $0 \leq\left|r_{22}-s_{22}\right|<\frac{|\operatorname{det} U|}{\mathrm{d}}$, so that $r_{22}=s_{22}$, i.e., $\left(\begin{array}{ll}r_{11} & r_{12} \\ r_{21} & r_{22}\end{array}\right)=\left(\begin{array}{ll}s_{11} & s_{12} \\ s_{21} & s_{22}\end{array}\right)$. This proves that any two elements in $\mathrm{J}_{2}$ are incongruent.

COROLLARY 3. Let $U \in \operatorname{Mat}_{2}(z)$ with $\operatorname{det} U \neq 0$. Then the cardinality of the complete residue system (mod. U) is $|\operatorname{det} U|^{2}$.

REMARK. If we consider the ring of $3 \times 3$ matrices, the corresponding results will read as follows, the proofs will be as in Lemma 3 and Theorem 2, with possible minor changes.

LEMMA 4. Let $u=\left(u_{i j}\right) \varepsilon \operatorname{Mat}_{3}(Z)$ with $\operatorname{det} U \neq 0$. Then

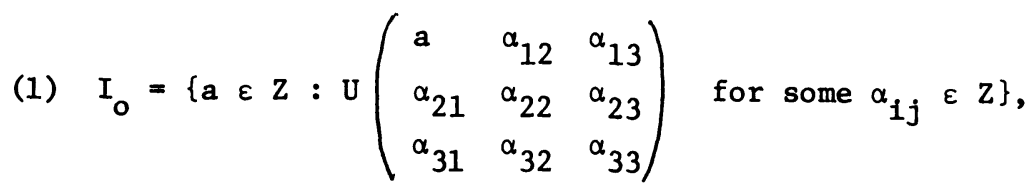




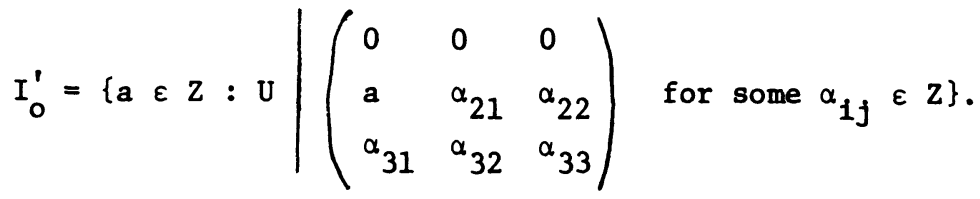

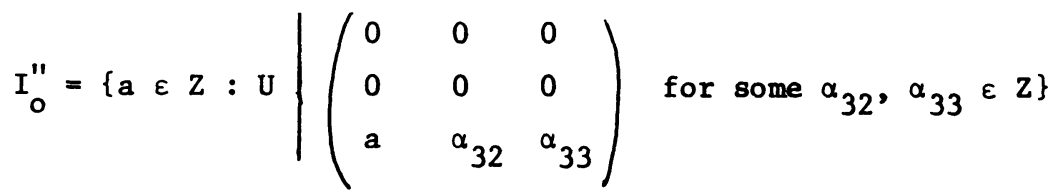

are nonzero principal ideals of $Z$ generated by the positive integer $g_{0}=$ g.c.d. $\left(u_{11}, u_{21}, u_{31}\right)$. Moreover, $I_{0}=I_{0}^{\prime}=I_{0}^{\prime \prime}$.

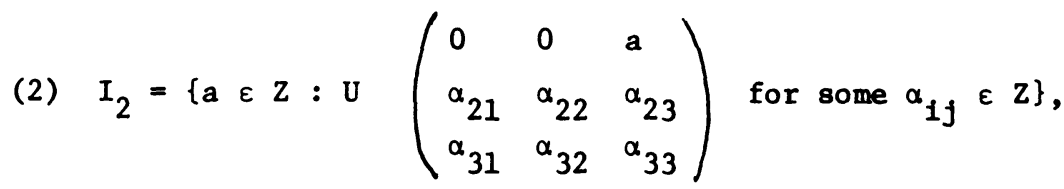

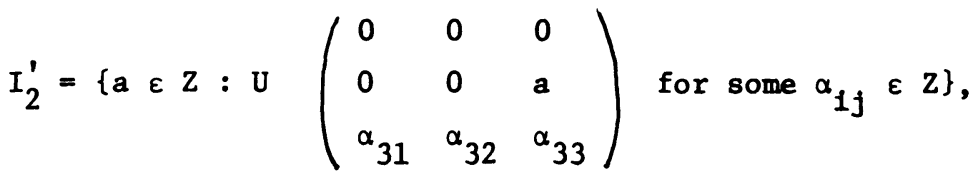

$$
\begin{aligned}
& I_{2}^{\prime \prime}=\left\{a \quad \varepsilon \quad Z: U\left(\begin{array}{lll}
0 & 0 & 0 \\
0 & 0 & 0 \\
0 & 0 & a
\end{array}\right)\right\}
\end{aligned}
$$

are nonzero principal ideals of $\mathrm{Z}$ generated by the positive integer $g_{2}=\frac{|\operatorname{det} U|}{g^{\prime}}$, where $g^{\prime}=g \cdot c \cdot d \cdot\left(\operatorname{cof}_{13}, \operatorname{cof} u_{23}, \operatorname{cof} u_{33}\right)$, and $\operatorname{cofu}_{1 j}$ is the cofactor of the element $u_{1 j}$. Moreover, $I_{2}=I_{2}^{\prime}=I_{2}^{\prime \prime}$.

(3) $I_{1}=\left\{\begin{array}{lllll}a & \varepsilon & Z & : & U\end{array} \mid \begin{array}{lll}0 & a_{1} & \alpha_{13} \\ \alpha_{21} & \alpha_{22} & \alpha_{23} \\ \alpha_{31} & \alpha_{32} & \alpha_{33}\end{array}\right)$ for some $\left.\alpha_{1\}} \varepsilon Z\right\}$

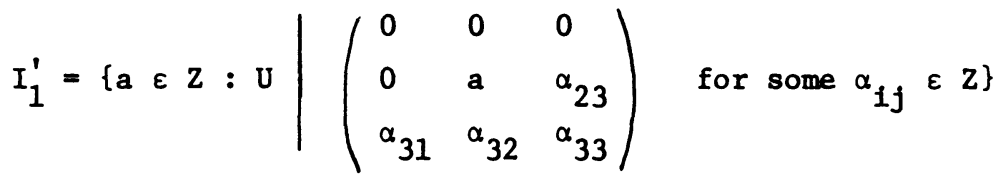

$$
\begin{aligned}
& \left.I_{1}^{\prime \prime}=\left\{\begin{array}{llll}
a & \varepsilon & Z & : U
\end{array} \mid \begin{array}{lll}
0 & 0 & 0 \\
0 & 0 & 0 \\
0 & 0 & \alpha_{33}
\end{array}\right) \quad \text { for some } \alpha_{33} \varepsilon z\right\}
\end{aligned}
$$


are nonzero principal ideals of $z$ generated by the positive integer $g_{1}=\frac{g^{\prime}}{g_{0}}$. Moreover, $I_{1}=I_{1}^{\prime}=I_{1}^{\prime \prime}$.

THEOREM 3. Let $U=\left(\begin{array}{lll}u_{11} & u_{12} & u_{13} \\ u_{21} & u_{22} & u_{23} \\ u_{31} & u_{32} & u_{33}\end{array}\right) \varepsilon \operatorname{Mat}_{3}(\mathrm{z}) \quad$ with $\operatorname{det} \neq 0$, let $g_{0}=g \cdot c \cdot d \cdot\left(u_{11}, u_{21}, u_{31}\right), g^{\prime}=g \cdot c \cdot d \cdot\left(\operatorname{cof} u_{13}, \operatorname{cof} u_{23}, \operatorname{cof} u_{33}\right)$. Then $J_{3}=\left\{R=\left[r_{1 j}\right] \& \operatorname{Mat}_{3}(Z): 0 \leq r_{1 j}<8_{j-1} \quad 1, j=1,2,3\right\} \quad$ is a complete residue system (mod. U) where $g_{1}=\frac{g^{\prime}}{g_{0}}, g_{2}=\frac{|\operatorname{det} U|}{8^{\prime}}$.

\section{REFERENCE}

1. Jordan, J. H. and C. J. Potratz. Complete Residue Systems in the Gaussian Integers, Math. Mag. 38 (1965) 1-12. 


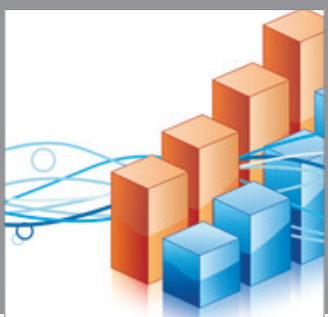

Advances in

Operations Research

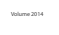

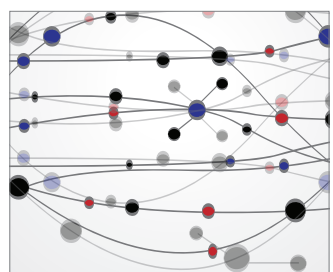

\section{The Scientific} World Journal
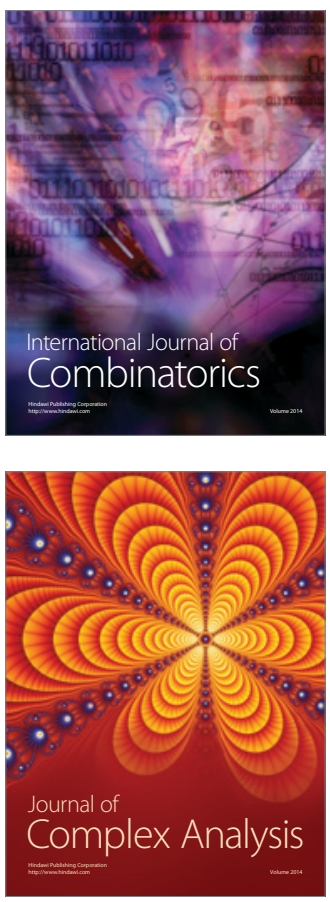

International Journal of

Mathematics and

Mathematical

Sciences
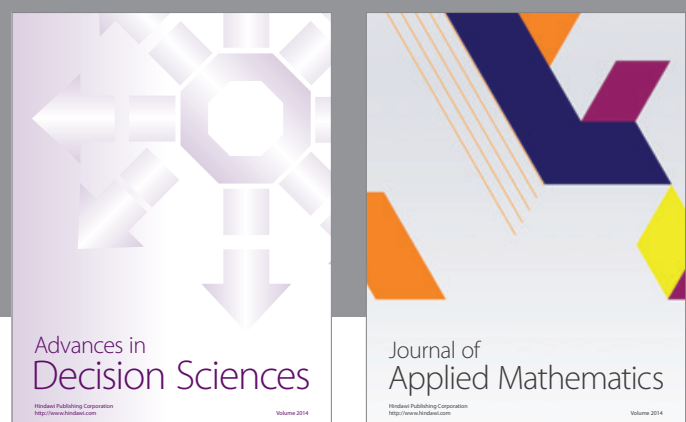

Journal of

Applied Mathematics
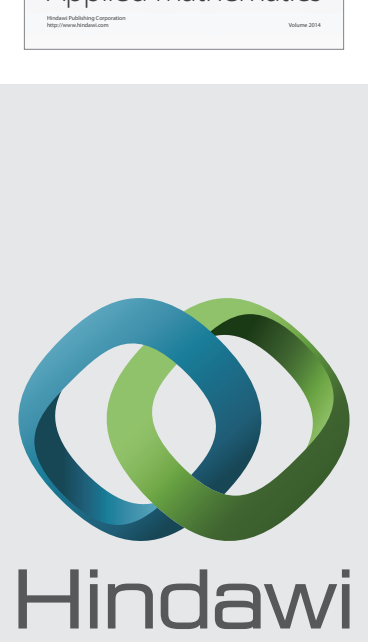

Submit your manuscripts at http://www.hindawi.com
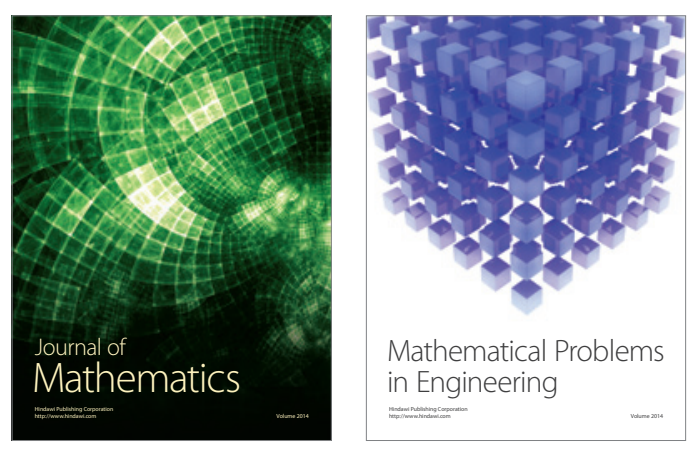

Mathematical Problems in Engineering
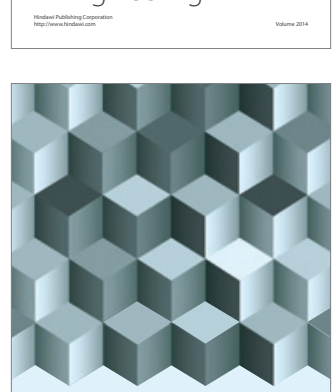

Journal of

Function Spaces
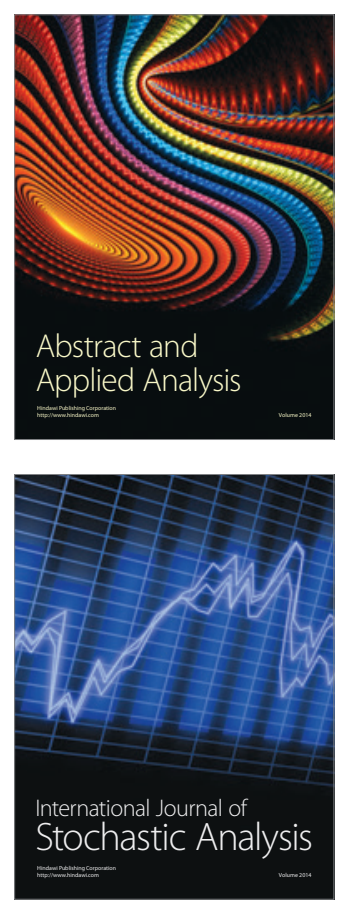

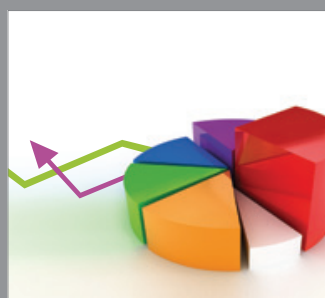

ournal of

Probability and Statistics

Promensencen
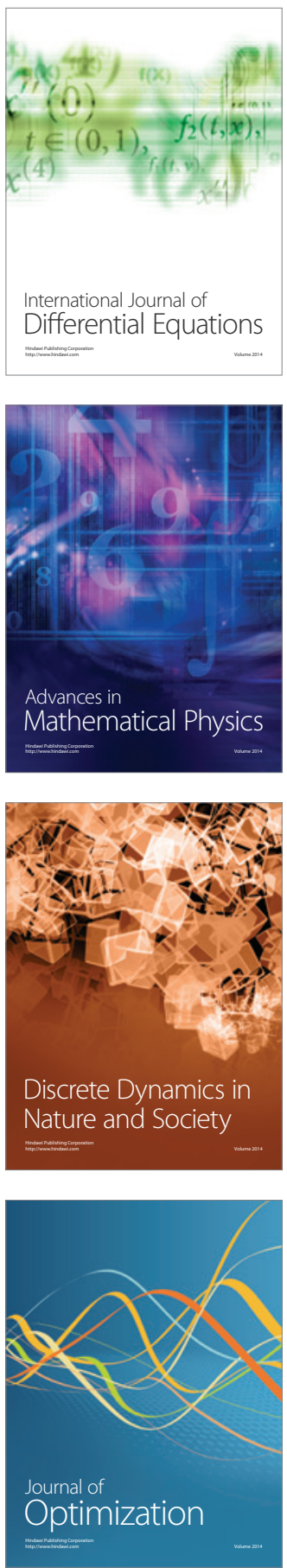